\title{
Efecto comparativo de nuevo colutorio-gel de clorhexidina con colutorios comerciales en el crecimiento de placa en 24 horas
}

\author{
RIVERA S * \\ YÉVENES I ** \\ REYES J *** \\ NORERO $\mathrm{H} * * * *$
}

\begin{abstract}
Rivera S, Yévenes I, Reyes J, Norero H. Efecto comparativo de nuevo colutorio-gel de clorhexidina con colutorios comerciales en el crecimiento de placa en 24 horas. Av Periodon Implantol. 2006; 18, 3: 163-169.
\end{abstract}

\section{RESUMEN}

Introducción. Las soluciones de clorhexidina (CHX), estándares en prevención de formación de placa presentan algunos efectos indeseados como el dolor por alcohol.

Objetivos. El propósito de este estudio fue determinar la eficacia clínica de colutorio-gel acuoso de clorhexidina al 0,1\% y compararla con colutorios al $0,12 \%$ y $0,1 \%$ con alcohol, evaluando el crecimiento de placa bacteriana.

Materiales y métodos. En un estudio cruzado, doble ciego y randomizado, 25 alumnos de buena salud oral, participaron de cuatro estudios con abstención de limpieza oral por 24 horas, separados entre si por 4 semanas. Los alumnos se enjuagaron por una vez con uno de los siguientes colutorios: CHX 0,12\% + alcohol (Oralgene ${ }^{\circledR}$ ), CHX 0,12\% + alcohol (Perio.Aid ${ }^{\circledR}$ ), CHX 0,1\%+ alcohol (Dentilim ${ }^{\circledR}$ ) y CHX 0,1\% + HMC 2,5\% (Colutoriogel ${ }^{\circledR}$, acuoso nueva formula). Después de un día se midió formación de placa, se completo y registro el cuestionario de parámetros clínicos.

Resultados. Las formulaciones de CHX 0,12\% + alcohol y CHX 0,1\% + HMC 2,5\% se mostraron eficientes en retardar el crecimiento de placa dental de novo, esto obtenido de las mediciones clínicas, y siempre superior $(\mathrm{p}<0,05)$ a la formulación del colutorio de CHX $0,1 \%+$ alcohol $\left(\right.$ Dentilim $^{\circledR}$ ).

Conclusiones. Los resultados de este estudio demuestran el potencial clínico de este nuevo colutorio-gel sin alcohol de CHX 0,1\% + HMC 2,5\% (Colutoriogel $尺$ ) como un efectivo agente antiplaca y con reducidas efectos secundarios registrados.

\section{PALABRAS CLAVE}

Clorhexidina, colutorio-gel, índice placa, colutorios.

Aceptado para publicación: Enero 2006.

* Profesor Asociado de Periodoncia. Dpto. de Odontología Conservadora. Facultad Odontología. Universidad de Chile.

** Profesor Asistente de Química. Dpto. de Ciencias Físicas y Químicas. Facultad Odontología. Universidad de Chile.

*** Químico. Dpto. de Ciencias Físicas y Químicas. Facultad Odontología. Universidadde Chile.

**** Cirujano Dentista. Dpto. de Odontología Conservadora. Facultad Odontología. Universidad de Chile. 


\section{INTRODUCCIÓN}

El control de la placa que es la eliminación de la placa microbiana y la prevención de su acumulación sobre dientes y superficies gingivales adyacentes, representa un mecanismo importante en el proceso de prevención de la caries dental y de las enfermedades periodontales. Además del control mecánico de la placa, se cuenta con inhibidores químicos, donde el más estudiado es la clorhexidina, sustancia aceptada por el Consejo de Terapéutica Dental de la ADA, y que en los seres humanos, no ha presentado ninguna evidencia de actividad tóxica ni resistencia de los microorganismos bucales. Ésta es una molécula diguanidohexano con propiedades antisépticas, retenida en la cavidad oral y liberada lentamente, asegurando un ambiente antibacteriano prolongado tras una sola aplicación. Su mecanismo de retención más importante es la interacción electrostática entre grupos cargados negativamente presentes en mucosa oral, placa y dientes y moléculas de clorhexidina cargada positivamente. Se ha demostrado que la placa retiene cantidades significativas de clorhexidina y otras sustancias catiónicas, y la aplicación de clorhexidina produce una inhibición en el desarrollo de la placa microbiana por sus efectos bacteriostáticos y bactericidas. Los estudios clínicos con clorhexidina de algunos meses de duración señalan una disminución de la placa y de la gingivitis $(1,2)$.

Las diferentes formulaciones de clorhexidina como colutorios en concentraciones de $0,12 \%, 0,2 \%$ y $2 \%$, barnices con concentraciones de 10 a $20 \%$, dentífricos en forma de gel en concentraciones al $1 \%$ y el chip de clorhexidina, indica la búsqueda de los investigadores de incrementar la cantidad de clorhexidina en los sitios de acción de ésta, pero normalmente asociado a un incremento en la concentración real de clorhexidina (3). Las soluciones de clorhexidina (CHX) al 0,2\%, están clasificadas como colutorios estándares en la prevención de formación de placa y desarrollo de la gingivitis, presentan algunos efectos indeseados como su coloración (café o negra) extrínseca de los dientes, mal sabor y alteraciones del sabor (en especial lo salado), cambios de sensibilidad de la lengua, y dolor por su contenido de alcohol (4). Estos efectos adversos han llevado al desarrollo de nuevas formulaciones de colutorios de clorhexidina de menor concentración y sin alcohol.

\section{PROPÓSITO}

El propósito de este estudio fue determinar la eficacia clínica de colutorio-gel acuoso de clorhexidina al 0,1\% elaborado por el laboratorio de Química de la Facultad de Odontología y compararla con colutorios al $0,12 \%$ y $0,1 \%$ con alcohol del mercado nacional a través de evaluaciones sobre el crecimiento de placa bacteriana.

\section{MATERIALES Y MÉTODOS}

Este estudio doble ciego, randomizado y cruzado involucro a 25 alumnos de la Facultad de Odontología con periodonto sano, quienes no utilizaron ningún medio de control de placa durante 4 periodos experimentales de un día (separados uno del otro por un periodo de descanso de 4 semanas). Durante cada periodo experimental, ellos se enjuagaron con uno de los siguientes enjuagatorios en una secuencia randomizada: CHX 0,12\% + alcohol (Oralgene ${ }^{\circledR}$ ), CHX 0,12\% + alcohol (Perio.Aid ${ }^{\circledR}$ ), CHX 0,1\%+ alcohol $\left(\right.$ Dentilim $^{\circledR}$ ) and CHX 0,1\% + HMC 2,5\% (Colutoriogel ${ }^{\circledR}$, nueva formulación sin alcohol. Los alumnos seleccionados según los criterios del estudio completaron un cuestionario de salud dental y un formulario de consentimiento para participar en el estudio. Un examen clínico oral y una evaluación clínica periodontal (vitalidad, sensibilidad dental, índice gingival, unión gingival y tejido blando) fue realizada a los participantes del estudio antes y después de la aplicación del colutorio. Los alumnos fueron asignados randomizadamente a los grupos de estudio y de control, con 20 alumnos en el grupo experimental y 5 en el grupo control. En tres de los periodos experimentales, el grupo control uso un colutorio sin clorhexidina y el grupo de estudio uso el colutorio con clorhexidina, en el cuarto estudio se utilizaron el colutorio-gel con y sin clorhexidina respectivamente. Alumnos sin gingivitis o solo con niveles bajos medidos por el índice Loe-Silness para gingivitis (GI) (5) participaron del estudio. Cada alumno recibió previo al uso de los colutorios indicaciones con respecto a su aseo bucal. Información acerca del objetivo del experimento e instrucciones escritas fueron entregadas a cada voluntario. Además se llevo un sistema de registro individual para registrar sensibilidad dental, irritación gingival y otros comentarios con respecto al uso de los colutorios.

\section{Procedimiento}

En la mañana de cada estudio, los estudiantes se lavaron con un dentífrico estándar y agua por dos minutos. Los dientes fueron coloreados con eritrosina y la placa residual fue removida con copa de goma sin pasta 
de pulir. Los estudiantes se enjuagaron con $10 \mathrm{ml}$ por 3 minutos con el enjuagatorio entregado bajo la supervisión de un investigador. La higiene oral fue suspendida por 24 horas y la placa acumulada en ese periodo fue revelada con eritrosina, cuantificando las áreas de superficies bucales tanto superiores como inferiores de incisivos, caninos y premolares usando el índice de placa de (6). Los pacientes seleccionados no usaron ningún producto con clorhexidina o derivados por lo menos 10 días antes de la experiencia y además, deberán tener una higiene normal para realizar los otros estudios con los otros productos de la investigación. En todos los estudios se usó un enjuagatorio control sin clorhexidina. Los estudios fueron separados por un período de 4 semanas.

Análisis estadístico. Los datos de índice de placa de placa entre los grupos de estudio fueron sometidos al test de Wilcoxon para datos no-paramétricos, para la comparación de datos interestudios se usó el test de Kruskal-Wallis y el t-Test para Muestras Pareadas del Índice de Placa (7).

Calibración de examinadores. Los examinadores completaron una sección de calibración en índice de placa e índice gingival previo al inicio del estudio.

\section{Determinación de clorhexidina en colutorios y colu-} torio-gel. Fue realizada por cromatografía liquida HPLC fijando el detector a $254 \mathrm{~nm}$ (8).

Envase de los colutorios. Los productos utilizados fueron envasados en frascos idénticos con un número identificador no conocido por el examinador.

\section{RESULTADOS}

No existieron signos clínicos de cambios en las mucosas después del uso de los diferentes colutorios. La mayoría de los voluntarios indico tener la sensación del colutorio en boca por un tiempo de 45 minutos aproximadamente. No se cuantifico la respuesta al sabor del colutorio, pero no se describieron alteraciones mayores al momento de usar salvo las descritas en la literatura por algunos participantes, no describiéndose estas para el colutoriogel.

Los resultados obtenidos para el índice de placa usando el índice de placa de Addy et al (1989) (6) pueden observarse en la tabla 1. En ella aparecen los resultados promedios de índice de placa para los pacientes antes del uso de los colutorios con clorhexidina y des- pués del uso de cada uno de ellos. Existen diferencias significativas entre el promedio de índice de placa medido al inicio de la experimentación (higiene oral usual) y los obtenidos solo con el uso del colutorio, sin higiene $(p<0,01)$. Al graficar índice de placa promedio y uso de colutorios con clorhexidina puede observarse la similitud de respuesta en el control de placa de los colutorios al 0,12\% y el colutorio gel, el menor índice de placa se observa al inicio de la experimentación (Fig. 1). Los resultados obtenidos con los colutorios controles sin clorhexidina no muestran diferencias entre ellos pero si hay diferencias significativas entre los colutorios controles y la medición inicial de placa promedio $(\mathrm{p}<0,001)$ (Tabla 2$)$. Al comparar los resultados entre los colutorios controles y los colutorios con clorhexidina se observa en todos diferencias significativas $(p<0,001)$ lo que señala efectividad de todas las formulaciones con clorhexidina (Fig. 2).

En la tabla 3 aparecen los resultados del estudio estadístico donde se comparan los índice de placa promedio interestudio entre los diferentes colutorios usados a través del $t$-test para muestras pareadas observándose diferencias significativas $(p<0,05)$ solo entre el colutorio 1 al $0,12 \%$ y el colutoriogel al $0,1 \%$ con respecto al colutorio 3 al 0,1\%. Al realizar el mismo $t$-test para muestras pareadas pero usando la raíz cuadrada del índice de placa (9) obtenido para los cuatro colutorios (Tabla 4), se observan diferencias significativas $(p<0,05)$ entre las formulaciones de clorhexidina al $0,12 \%$ ( 1 y 2 ) y el colutoriogel con respecto al colutorio al $0,1 \%$ en clorhexidina.

Las formulaciones de clorhexidina al 0,12\% con alcohol y la del colutoriogel de clorhexidina al 0,1\% mas $2 \%$ de HMC tuvieron igual efectividad en retardar el crecimiento de placa, la de menor efectividad correspondió a la formulación de clorhexidina al 0,1\% con alcohol (Fig. 2).

\section{DISCUSION}

La información sobre el control químico de la placa microbiana ha sido revisada por varios autores confirmándose que la clorhexidina, en enjuagatorios, es el más efectivo agente antiplaca documentado (10), además, es una sustancia de alta eficiencia en el control de patógenos periodontales (11). La eficacia clínica de la clorhexidina parece estar asociada a la adsorción de este antiséptico a superficies orales a través de fuerzas electrostáticas. Esta adsorción permitiría explicar la persistente actividad antimicrobiana de varias ho- 


\begin{tabular}{|c|c|c|c|c|c|}
\hline \multicolumn{6}{|c|}{$\begin{array}{l}\text { TABLA 1.- ÍNDICE DE PLACCA PROMEDIO INICIAL Y POSTERIOR AL USO DE } \\
\text { COLUTORIOS DE } \\
\text { CLORHEXIDINA }\end{array}$} \\
\hline Paciente & Inicial & $\begin{array}{c}\text { CHX } 0,12 \% \\
1\end{array}$ & $\begin{array}{c}\text { CHX } 0,12 \% \\
2\end{array}$ & $\begin{array}{c}\text { CHX } 0,10 \% \\
3\end{array}$ & $\begin{array}{c}\text { CHX-G } 0,10 \% \\
4\end{array}$ \\
\hline $\begin{array}{c}1 \\
2 \\
3 \\
4 \\
5 \\
6 \\
7 \\
8 \\
9 \\
10 \\
11 \\
12 \\
13 \\
14 \\
15 \\
16 \\
17 \\
18 \\
19 \\
20 \\
\text { Promedio } \\
\text { D.S. }\end{array}$ & $\begin{array}{l}0,3 \\
0 \\
0,1 \\
0 \\
0 \\
0,9 \\
0 \\
0 \\
0,4 \\
0 \\
0 \\
0 \\
0 \\
0 \\
0 \\
0 \\
0 \\
0 \\
0 \\
0 \\
0,084 \\
0,2159\end{array}$ & $\begin{array}{l}0,4 \\
0 \\
0,3 \\
0,2 \\
0,3 \\
0,9 \\
0,5 \\
1,2 \\
0,3 \\
0,2 \\
0 \\
0 \\
0,3 \\
0,3 \\
0 \\
0 \\
0,2 \\
0,5 \\
0 \\
0,8 \\
0,319 \\
0,328\end{array}$ & $\begin{array}{l}1 \\
0,3 \\
0 \\
0 \\
0 \\
0 \\
0 \\
0 \\
0 \\
0 \\
0 \\
0,4 \\
0,2 \\
0 \\
0,8 \\
0,1 \\
1,2 \\
1,5 \\
0,1 \\
0,4 \\
0,296 \\
0,456\end{array}$ & $\begin{array}{l}0 \\
0,9 \\
0,7 \\
0,7 \\
0,6 \\
0,5 \\
0,2 \\
1,4 \\
0,9 \\
0,2 \\
0,3 \\
0,4 \\
0,4 \\
0,4 \\
0,25 \\
0,3 \\
0,7 \\
1,3 \\
0 \\
0,4 \\
0,521 \\
0,376\end{array}$ & $\begin{array}{l}0,5 \\
0,1 \\
0,1 \\
0 \\
0,3 \\
0,9 \\
0,2 \\
0,1 \\
0,7 \\
0 \\
0,4 \\
0,2 \\
0 \\
0,8 \\
0,4 \\
0 \\
0 \\
1,1 \\
0 \\
0,4 \\
0,309 \\
0,337\end{array}$ \\
\hline
\end{tabular}

CHX 0,12\% 1: Colutorio de clorhexidina al 0,12\% con alcohol (Oralgene ${ }^{(\mathrm{R})}$ ); $C H X$ 0,12\% 2: Colutorio de clorhexidina al 0,12\% con alcohol $\left(\right.$ Perioaid $\left.^{(\mathrm{R})}\right)$; CHX 0,10\% 3: Colutorio de clorhexidina al 0,1\% con alcohol (Dentilim ${ }^{(\mathrm{R})}$ ); $C H X-G$ 0,1\% 4: Colutorio-gel de clorhexidina al 0,1\% sin alcohol mas $2 \%$ HMC; D.S.: desviación estándar.

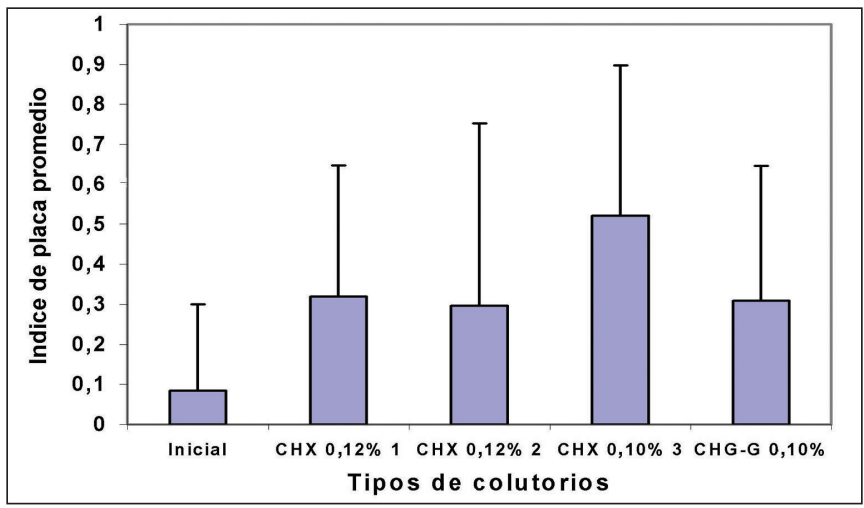

Fig. 1: Índice de placa promedio al inicio y posterior al uso de colutorios de clorhexidina. CHX 0,12\% 1: Colutorio de clorhexidina al $0,12 \%$ con alcohol (Oralgene $\left.{ }^{\circledR}\right)$; CHX 0,12\% 2: Colutorio de clorhexidina al 0,12\% con alcohol (Perioaid ${ }^{\circledR}$ ); CHX 0,10\% 3: Colutorio de clorhexidina al 0,1\% con alcohol (Dentilim $\left.{ }^{\circledR}\right)$; CHX-G 0,1\%: Colutorio-gel de clorhexidina al 0,1\% sin alcohol más $2 \%$ HMC. ras de la clorhexidina (12). Esta eficacia antiplaca de los diferentes colutorios con clorhexidina usados se demostró al comparar estos con colutorios controles sin clorhexidina, obteniéndose diferencias significativas en todos ellos. Nos llama la atención si que la inhibición del crecimiento de placa solo con el uso del colutorio (sin aseo bucal) sea inferior al estado basal, con el aseo habitual, lo que nos da cuenta de la importancia de este en el control de la placa microbiana, aunque el periodo sin higiene bucal sea tan solo de 24 horas.

Debido a la acción que ejerce la clorhexidina sobre la placa microbiana, se pueden realizar mediciones de áreas de placa e índice de placa que permiten cuantificar este efecto de la clorhexidina sobre el crecimiento de la placa en 24 horas, mediciones que permiten, con un cierto grado de certeza, ser usadas como métodos rápidos para evaluar agentes y formulaciones poten- 


\section{TABLA 2.- INDICE DE PLACA PROMEDIO INICIAL Y POSTERIOR AL USO DE COLUTORIOS CONTROLES SIN CLORHEXIDINA}

\begin{tabular}{|c|l|l|l|l|l|}
\hline \multirow{2}{*}{ Paciente } & \multirow{2}{*}{ Inicial } & \multicolumn{4}{|c|}{ Controles } \\
\cline { 3 - 6 } & & \multicolumn{1}{|c|}{$\mathbf{2}$} & \multicolumn{1}{|c|}{$\mathbf{3}$} \\
\hline 1 & 0,2 & 1,3 & 1,2 & 1,1 & 1,2 \\
2 & 0 & 0,65 & 0,75 & 0,45 & 0,6 \\
3 & 0 & 0,45 & 0,55 & 0,65 & 0,5 \\
4 & 0,25 & 1,28 & 1,3 & 1,2 & 0,9 \\
5 & 0 & 0,7 & 0,65 & 0,65 & 0,85 \\
Promedio & $\mathbf{0 , 0 9}$ & $\mathbf{0 , 8 7 6}$ & $\mathbf{0 , 8 9}$ & $\mathbf{0 , 8 1}$ & $\mathbf{0 , 8 1}$ \\
D.S. & $\mathbf{0 , 1 2 5}$ & $\mathbf{0 , 3 8 9}$ & $\mathbf{0 , 3 3 8}$ & $\mathbf{0 , 3 2 3}$ & $\mathbf{0 , 2 7 5}$ \\
\hline
\end{tabular}

Control 1: Colutorio sin clorhexidina con alcohol; Control 2: Colutorio sin clorhexidina con alcohol; Control 3: Colutorio sin clorhexidina con alcohol; Control 4: Colutorio-gel sin clorhexidina, sin alcohol más $2 \%$ HMC. D.S: desviación estándar.

cialmente antiplaca (13). A través de la medición del índice de placa fue posible comparar la efectividad del colutoriogel de clorhexidina con los tres colutorios de clorhexidina, dos al 0,12\% y uno al 0,1\%, donde el colutoriogel presento una respuesta equivalente a los colutorios al 0,12\% y superior al colutorio al 0,1\% en retardar el crecimiento de placa (Fig. 1 y Fig. 2), en esencia si comparamos la efectividad del colutoriogel con los colutorios al 0,12\% vemos que es mayor la del colutoriogel pues esta formulado al $0,1 \%$ en clorhexidina, por lo tanto igual efectividad pero obtenida con una menor concentración.

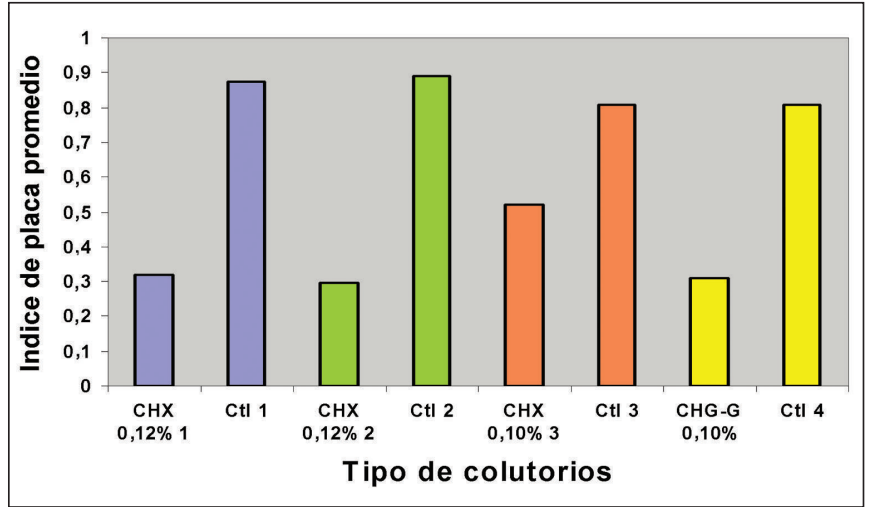

Fig. 2: Índice de placa promedio con colutorios con clorhexidina y control sin clorhexidina. CHX 0,12\% 1: Colutorio de clorhexidina al $0,12 \%$ con alcohol (Oralgene ${ }^{\circledR}$ ); CHX 0,12\% 2: Colutorio de clorhexidina al 0,12\% con alcohol (Perioaid ${ }^{\circledR}$; CHX 0,10\% 3: Colutorio de clorhexidina al 0,1\% con alcohol (Dentilim ${ }^{\circledR}$ ); CHX-G 0,1\%: Colutorio-gel de clorhexidina al 0,1\% sin alcohol mas $2 \% \mathrm{HMC}$; Ctl 1 : Colutorio sin clorhexidina mas alcohol; Ctl 2: Colutorio sin clorhexidina con alcohol; Ctl 3: Colutorio sin clorhexidina con alcohol; Ctl 4: Colutorio-gel sin clorhexidina, sin alcohol más 2\% HMC.

En la búsqueda de optimizar la captación y posterior entrega de clorhexidina por los elementos de la cavidad oral, desarrollamos en nuestro laboratorio un colutorio de clorhexidina en forma de gel, con características tixotrópicas, es decir, su viscosidad disminuye por agitación, para ser usado por los pacientes. La viscosidad juega un rol importante en esta formulación, pues no solo la estabiliza, sino que al disminuir su viscosidad por agitación, transformando el gel en una solución, facilita la difusión de la clorhexidina en la cavidad bucal, mientras que en reposo, la viscosidad de la solución

\begin{tabular}{|l|c|c|c|c|}
\hline \multicolumn{4}{|c|}{ TABLA 3.- $t$ TEST PARA MUESTRAS PAREADAS DEL ÍNDICE DE PLACA } \\
\hline & CHX 0,12\% 1 & CHX 0,12\% 2 & CHX 0,1\% 3 & CHX-G 0,1\% 4 \\
\hline CHX 0,12\% 1 & - & $t=0,176$ & $t=-2,446$ & $t=0,115$ \\
& $t=0,176$ & $\mathrm{P}=0,862$ & $\mathrm{P}=0,024^{*}$ & $\mathrm{P}=0,909$ \\
\hline CHX 0,12\% 2 & $\mathrm{P}=0,862$ & - & $t=-1,837$ & $t=-0,118$ \\
& $t=-2,446$ & $t=-1,837$ & $\mathrm{P}=0,082$ & $\mathrm{P}=0,907$ \\
\hline CHX 0,1\% 3 & $\mathrm{P}=0,024^{*}$ & $\mathrm{P}=0,082$ & $-2,140$ \\
& $t=0,115$ & $t=-0,118$ & $t=-2,140$ & $\mathrm{P}=0,046^{*}$ \\
\hline CHX-G 0,1\% & $\mathrm{P}=0,909$ & $\mathrm{P}=0,907$ & $\mathrm{P}=0,046 *$ & - \\
&
\end{tabular}

CHX 0,12\% 1: Colutorio de clorhexidina al 0,12\% con alcohol (Oralgene ${ }^{\circledR}$ ); $C H X$ 0,12\% 2: Colutorio de clorhexidina al 0,12\% con alcohol (Perioaid ${ }^{\circledR}$ ); CHX 0,10\% 3: Colutorio de clorhexidina al 0,1\% con alcohol (Dentilim ${ }^{\circledR}$ ); CHX-G 0,1\% 4: Colutorio-gel de clorhexidina al 0,1\% sin alcohol mas $2 \%$ HMC; *: Diferencias significativas. 


\begin{tabular}{|c|c|c|c|c|}
\hline \multicolumn{5}{|c|}{$\begin{array}{c}\text { TABLA 4.- } t \text { TEST PARA MUESTRAS PAREADAS DE IAA RÁlz CUADRADA } \\
\text { DEL ÍNDICE DE PLACA }\end{array}$} \\
\hline & CHX 0,12\% 1 & CHX 0,12\% 2 & CHX 0,1\% 3 & CHX-G 0,1\% 4 \\
\hline CHX 0,12\% 1 & - & $\begin{array}{l}t=0,713 \\
\mathrm{P}=0,483\end{array}$ & $\begin{array}{l}t=-2,406 \\
\mathrm{P}=0,024^{*}\end{array}$ & $\begin{array}{l}t=0,184 \\
\mathrm{P}=0,855\end{array}$ \\
\hline CHX 0,12\% 2 & $\begin{array}{l}t=0,713 \\
\mathrm{P}=0,483\end{array}$ & - & $\begin{array}{l}\mathrm{t}=-2,378 \\
\mathrm{P}=0,026^{*}\end{array}$ & $\begin{array}{l}t=-0,658 \\
\mathrm{P}=0,517\end{array}$ \\
\hline CHX 0,1\% 3 & $\begin{array}{l}t=-2,406 \\
\mathrm{P}=0,024^{*}\end{array}$ & $\begin{array}{l}t=-2,378 \\
\mathrm{P}=0,026^{*}\end{array}$ & - & $\begin{array}{l}t=-2,277 \\
P=0,032^{*}\end{array}$ \\
\hline CHX-G 0,1\% & $\begin{array}{l}t=0,184 \\
\mathrm{P}=0,855\end{array}$ & $\begin{array}{l}t=-0,658 \\
\mathrm{P}=0,517\end{array}$ & $\begin{array}{l}t=-2,277 \\
\mathrm{P}=0,032 *\end{array}$ & - \\
\hline
\end{tabular}

CHX 0,12\% 1: Colutorio de clorhexidina al 0,12\% con alcohol (Oralgene ${ }^{\circledR}$ ); $C H X$ 0,12\% 2: Colutorio de clorhexidina al 0,12\% con alcohol (Perioaid ${ }^{\circledR}$ ); CHX 0,10\% 3: Colutorio de clorhexidina al 0,1\% con alcohol (Dentilim ${ }^{\circledR}$ ); CHX-G 0,1\% 4: Colutorio-gel de clorhexidina al 0,1\% sin alcohol más 2\% HMC; *: Diferencias significativas.

aumenta, transformándose en un gel que permite un mayor tiempo de contacto de la clorhexidina con la pieza dentaria, placa microbiana y mucosas, con la clorhexidina cuando su viscosidad aumenta. La otra diferencia con los colutorios tradicionales es que el colutoriogel esta libre de alcohol, pues el gelificante, HMC, es hidrosoluble lo que permite la eliminación del alcohol de la formulación, junto con mejorar el sabor al enmascarar en parte el sabor de la clorhexidina. Las ventajas de este gel tixotrópico es que posee en parte las características de los colutorios en cuanto a dosificación y entrega de la clorhexidina (14) y las propiedades de los geles, de conveniente disponibilidad y almacenaje del producto. Estudios clínicos indican que una mejor eficacia terapéutica se puede alcanzar usando los geles para las infecciones orales que los tratamientos convencionales que usan enjuagatorios (15).

El desarrollo de este colutorio-gel de clorhexidina al $0,1 \%$ permitirá su uso en programas masivos de prevención de control de placa microbiana con una concentración un $20 \%$ menor que los colutorios existentes en el mercado farmacéutico con igual o mejor efectividad.

\section{SUMMARY}

The chlorhexidine solutions ( $\mathrm{CHX}$ ) standars in prevention of plaque formation present some undesired effects like the pain by alcohol.

The aim of this study was to determine the clinical effectiveness of Colutoriogel ${ }^{\circledR}$ (new formulation) of chlorhexidine to the $0,1 \%$ and to compare with mouthrinses to the $0,12 \%$ and $0,1 \%$ with alcohol being evaluated growth of bacterial plaque.

In double-blind, randomised, cross-over study, 25 young dental students with a healthy periodontium, abolished all means of mechanical plaque control during 4 experimental periods of 1 day (separated from each other by a washout period of 4 weeks). During each experimental period, they rinsed 1 daily with one of the following mouthrinses in a randomised order: CHX 0,12\% + alcohol (Oralgene ${ }^{\circledR}$ ), CHX 0,12\% + alcohol (Perio.Aid $\left.{ }^{\circledR}\right)$, CHX 0,1\%+ alcohol (Dentilim ${ }^{\circledR}$ ) and $\mathrm{CHX} 0,1 \%+\mathrm{HMC} 2,5 \%\left(\right.$ Colutoriogel $\left.^{\circledR}\right)$. After 1 days of undisturbed plaque formation, clinical parameters were recorded and questionnaires completed.

The CHX 0,12\% + alcohol and the and CHX 0,1\%+ HMC 2,5\% formulations were efficient in retarding de novo plaque formation (proven by clinical observations data), and always superior $(p<0,05)$ to the CHX $0,1 \%+$ alcohol (Dentilim ${ }^{\circledR}$ ) solution.

Conclusions. The results of this study demonstrated the potential of a new mouthrinses-gel Colutoriogel ${ }^{\circledR}$ ) nonalcoholic formulation as an effective anti-plaque agent with reduced unpleasant subjective side-effects.

\section{KEY WORDS}

Chlorhexidine, mouthrinses-gel, plaque index, mouthrinses. 


\section{CORRESPONDENCIA}

Prof. I.Yébenes López

Facultad de Odontología

Olivos 943 Independencia

Santiago, Chile

E-mail: iyevenes@odontologia.uchile.cl

\section{BIBLIOGRAFIA}

1. Charles CH. Mostler KM. Bartels LL. Mankodi SM. Comparative antiplaque and antigingivitis effectiveness of a chlorhexidine and an essential oil mouthrinse: 6month clinical trial. J Clin Periodontol 2004; 31(10): 878-84.

2. Clavero J. Baca P. Paloma Gonzalez M. Valderrama MJ. Efficacy of chlorhexidine-thymol varnish (Cervitec) against plaque accumulation and gingival inflammation in a geriatric population. Gerodontology 2006; 3(1): 43-7.

3. Paraskevas S. Randomized controlled clinical trials on agents used for chemical plaque control. Int J Dent Hyg 2005;(4):162-78.

4. Addy M. Moran JM. Clinical indications for the use of chemical adjuncts to plaque control: chlorhexidine formulations. Periodontol 2000 1997;15: 52-4.

5. Loe $H$. The gingival index, the plaque index and the retention index systems. J. Periodontol 1967;38 (Supplement): 610-6.

6. Addy M. Wade W. Jenkins S. Goodfield S. Comparison of two commercially available chlorhexidine I. Staining and antimicrobial effects in vitro. Clin Prev Dent 1989; 11: 10-4.
7. Schlesselman J.: Monographs in epidemiology and Biostatistics. Caso - Control Studies. Oxford University, 1998.

8. Yévenes I. Reyes J. Norero H. Monardes V. Rivera S. Efecto inhibitorio en placa microbiana y propiedades antibacterianas de enjuagatorios de clorhexidina. Av. Periodon. Implantol 2003; 15(1):19-24.

9. Fleis JL. Statical transformations of indices of gingivitis measured non-invasively. J Clin Periodontol 1985; 12 : 750-5.

10. Twetman S. Antimicrobials in future caries control? A review with special reference to chlorhexidine treatment. Caries Res 2004; 38(3): 223-9.

11. Perinetti G. Paolantonio M. Cordella C. D'Ercole S. Serra E. Piccolomini R. Clinical and microbiological effects of subgingival administration of two active gels on persistent pockets of chronic periodontitis patients. J Clin Periodontol 2004; 31(4): 273-81.

12. Rosenthal S. Spangberg L. Safavi K. Chlorhexidine substantivity in root canal dentin. Oral Surg Oral Med Oral Pathol Oral Radiol Endod. 2004. 98(4): 488-92.

13. Claydon N. Addy M. The use of plaque area and plaque index to measure the effect of fluoride and chlorhexidine toothpastes on 24 -h plaque regrowth. J Clin Periodontol 1995; 22: 540-2.

14. Fejerskov O. Ekstrand J. Burt BA. Fluoride in Dentistry. Ed. Munksgaard, Copenhagen 2a Ed. pag. 311-27; 1996.

15. Pai MR. Acharya LD. Udupa N. The effect of two different dental gels and a mouthwash on plaque and gingival scores: a six-week clinical study. Int Dent J. 2004; 54(4): 219-23. 